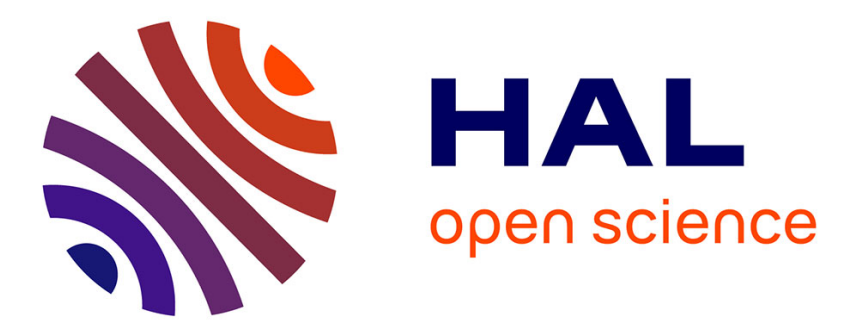

\title{
Influence of monomer reactivity on radiation grafting of phosphorus flame retardants on flax fabrics
}

Raymond Hajj, Belkacem Otazaghine, Rodolphe Sonnier, Roland El Hage, Sophie Rouif, Michel Nakhl, José-Marie Lopez-Cuesta

\section{- To cite this version:}

Raymond Hajj, Belkacem Otazaghine, Rodolphe Sonnier, Roland El Hage, Sophie Rouif, et al.. Influence of monomer reactivity on radiation grafting of phosphorus flame retardants on flax fabrics. Polymer Degradation and Stability, 2019, 166, pp.86-98. 10.1016/j.polymdegradstab.2019.05.025 . hal-02425052

\section{HAL Id: hal-02425052 \\ https://hal.science/hal-02425052}

Submitted on 3 Jun 2021

HAL is a multi-disciplinary open access archive for the deposit and dissemination of scientific research documents, whether they are published or not. The documents may come from teaching and research institutions in France or abroad, or from public or private research centers.
L'archive ouverte pluridisciplinaire HAL, est destinée au dépôt et à la diffusion de documents scientifiques de niveau recherche, publiés ou non, émanant des établissements d'enseignement et de recherche français ou étrangers, des laboratoires publics ou privés. 


\title{
Influence of monomer reactivity on radiation grafting of phosphorus flame retardants on flax fabrics
}

\author{
Raymond Hajj ${ }^{\text {a, b, c }}$, Belkacem Otazaghine ${ }^{\text {b }}$, Rodolphe Sonnier ${ }^{\text {b, * }}$, Roland El Hage ${ }^{\text {a, c, **, }}$ \\ Sophie Rouif ${ }^{d}$, Michel Nakhl ${ }^{\text {a, c }}$, José-Marie Lopez-Cuesta ${ }^{b}$ \\ ${ }^{a}$ LCPM, Faculty of Sciences, Lebanese University, Fanar, Lebanon \\ b C2MA, IMT - Mines Alès, 6, avenue de Clavières, 30100, Alès, France \\ ${ }^{c}$ Plateforme de Recherche en Nanosciences et Nanotechnologies, Lebanese University, EDST, Lebanon \\ d Ionisos SA, Dagneux, France
}

\begin{abstract}
A B S T R A C T
Flax fabrics were modified by radiation grafting method to improve their flame retardancy. Several phosphonated monomers with different carbon-carbon double bond reactivity were grafted. According to ${ }^{1} \mathrm{H}$ NMR carried out on irradiated monomers solubilized in water, the reactivity is the highest for (acryloyloxy)methyl phosphonic acid (hPAAPC1), and the lowest for allyl phosphonic acid (APA). Nevertheless, grafting yield on flax, assessed by X-ray fluorescence and scanning electron microscopy showed a different tendency. Especially (methacryloyloxy)methyl phosphonic acid (hPMAPC1) and vinyl phosphonic acid (VPA) appear to be highly grafted. In all cases, diffusion of the molecules into the flax elementary fibers bulk was observed. The choice of the solvent in washing step after the irradiation step showed to be effective to control the final phosphorus content in flax fabrics. The effect of phosphorus grafting on thermal properties and fire behavior was studied using thermogravimetric analysis, pyrolysis combustion flow calorimetry and a preliminary fire test. According to the latter, self-extinguishing and non-flammable fabrics were obtained for phosphorus content of $0.5 \mathrm{wt} \%$ and $1.2 \mathrm{wt} \%$ respectively. Correlations with flammability at microscale were pointed out. Additional cone calorimeter tests highlight the influence of flame retardant treatment on flammability under forced flaming conditions.
\end{abstract}

Keywords:

Flame retardancy

Flax

Phosphorus

Grafting

E-beam radiation

Reactivity

\section{Introduction}

Textile industry has been interested for centuries in natural fibers (especially cotton and flax) as main resources for their products manufacturing [1]. It has been reported that these fibers present many advantages like renewability, biodegradability, good availability, low cost, low density, non-toxicity and good mechanical properties [2-4]. Nevertheless, they also present many disadvantages as their flammability [5-8].

Natural fibers are mainly composed of cellulose, hemicelluloses and lignin [9]. It has been reported that natural fibers with low

\footnotetext{
* Corresponding author. C2MA, IMT - Mines Alès, 6, avenue de Clavières, 30100, Alès, France

** Corresponding author. LCPM, Faculty of Sciences, Lebanese University, Fanar, Lebanon.

E-mail addresses: rodolphe.sonnier@mines-ales.fr (R. Sonnier), roland_hag@ul. edu.lb (R. El Hage).
}

cellulose (less than 49\%) and high lignin content (more than 23\%) as coir and deciduous wood fibers have lower flammability behavior than, for example, cotton with higher cellulose (85-90\%) content [6].

Many methods have been investigated to improve flame retardancy such as UV irradiation [10,11], plasma treatment [12], sol-gel treatment [13], gamma or e-beam irradiation [14-17] in order to achieve chemical coupling after impregnation of flame retardants (FRs).

Many studies have focused their research on phosphoruscontaining flame-retardants (FRs) [10,18-27] since halogencontaining FRs have many concerns as releasing toxic gases during burning $[6,28]$.

Horrocks and Zhang [29] grafted high content of phosphorus (2.47 wt\%) onto cotton using chemical modification. Spyrocyclic pentaerythritol di(phosphonyl chloride) was dissolved in dimethylformamide. The authors proved that the phosphorylation of cellulose was achieved after heating the mixture at $160^{\circ} \mathrm{C}$ during 
$4 \mathrm{~h}$. At lower temperatures, phosphorus grafting was much lower. However, a temperature of $160^{\circ} \mathrm{C}$ could be considered as too high since it might be degradant for natural fibers.

Dorez et al. [30] grafted phosphorus onto flax fibers using octadecylphosphonic acid (ODPA) in ethanol/water (90/10 wt\%). The mixture was heated under reflux (around $80^{\circ} \mathrm{C}$ ) during $5 \mathrm{~h}$. The authors assumed that phosphonic function has high reactivity only with the aromatic hydroxyl groups from lignin in such conditions. Results showed that only low phosphorus content ( $0.5 \mathrm{wt} \%$ ) can be grafted onto flax fibers due to the low-lignin composition of flax.

Radiation grafting was also used to impart flame retardancy on flax fabrics [15-17]. The method is based on an impregnation step coupled to electron beam irradiation which is called mutual radiation grafting. Sonnier et al. [15] modified flax fabrics by reacting with dimethylvinyl phosphonate (MVP) and dimethyl(methacryloxy) methyl phosphonate (MAPC1) using radiation grafting. MVP was grafted into the bulk of the elementary fiber whereas MAPC1 was located on the surface only. Phosphorus content reached $4 \mathrm{wt} \%$ after dipping flax fabrics in a solution of $10 \mathrm{wt} \%$ MVP in THF, and irradiating them at $50 \mathrm{kGy}$. Self-extinguishing fabrics were prepared with only $1.5 \mathrm{wt} \%$ of phosphorus.

Hajj et al. [16] compared chemical modification and radiation grafting using vinyl phosphonic acid (VPA). Contrarily to other studies VPA as flame retardant [31,32], no initiator was used. In chemical modification, fabrics were dipped in various solutions of VPA with different concentrations and treated in different conditions where temperature values ranged between 70 and $160^{\circ} \mathrm{C}$ for $2 \mathrm{~h}$. Chemical modification in less severe conditions was not effective. The phosphorus grafted amount was very low due to lowlignin content of the flax fabrics. Chemical modification was much more interesting in severe conditions (DMF $-160^{\circ} \mathrm{C}$ ) where the final phosphorus content reached $2.3 \mathrm{wt} \%$. In these conditions, VPA phosphorylated the cellulose which constitutes $81 \mathrm{wt} \%$ of the flax fabrics. However, these conditions were considered as very degradant to the flax fibers. Radiation grafting was more successful as a grafting route of VPA on flax fabrics. Phosphorus content reached $1.4 \mathrm{wt} \%$ using a $10 \mathrm{wt} \%$ of an aqueous VPA solution and a radiation dose of $50 \mathrm{kGy}$. This P-content was sufficient to obtain self-extinguishing fabrics. As a conclusion, radiation grafting method was considered as more suitable to natural fibers that contains low lignin content.

Teixeira et al. [17] compared the grafting of phosphorus, bromine and sulfonamide-containing FRs using radiation grafting. According to the authors, the success of this method depends on a fast diffusion of the FR into the fibers, a low volatilization during the period between impregnation and irradiation and a high grafting efficiency. However, bromine and sulfonamide FRs grafting was negligible. Radiation grafting does not seem to be suitable for FR molecules acting as flame inhibitor (i.e. as radical scavenger). Phosphorus-containing FR showed to be the most efficient among the tested ones. However, the reactivity of the carbon-carbon double bond depends on the FR structure and this point must be further investigated.

In this paper, flax fabrics were treated using different phosphonated monomers to study the reactivity of the carbon-carbon double bond under irradiation. In order to better understand the grafting mechanism, the reactivity of different acrylate, methacrylate, vinyl and allyl phosphonate monomers was evaluated under ebeam radiation. Indeed, the reactivity of these functions is strongly different in radical polymerization. Acrylate and methacrylate are the most reactive functions and allyl is the least reactive one. Therefore various grafting yields are expected. Many factors influencing the radiation grafting process were also studied. For this, fabrics impregnated with the different monomers were irradiated at different doses to assess the influence of the radiation dose. For this procedure, no additives were used to promote monomers reactivity. Also, some of the treated fabrics were washed with two different solvents to reveal the importance of the solvent in order to control the final phosphorus content. Finally, flame retardancy of modified flax fabrics was assessed.

\section{Experimental part}

\subsection{Materials}

Flax fabrics (twill weave $2 \times 2$ ) were kindly provided by Hexcel (France). Fabric weight is $200 \mathrm{~g} / \mathrm{m}^{2}$. The chemical composition of the used flax fabric is $81 \mathrm{wt} \%$ of cellulose, $13 \mathrm{wt} \%$ of hemicelluloses and $2.7 \mathrm{wt} \%$ of lignin [15].

(Acryloyloxy)methyl phosphonic acid (hPAAPC1), (Methacryloyloxy)methyl phosphonic acid (hPMAPC1), Dimethyl(methacryloyloxy)methyl phosphonate (MAPC1), Allyl phosphonic acid (APA), Vinyl phosphonic acid (VPA) purchased from Specific Polymers (Castries, France) and dimethylvinyl phosphonate (MVP) purchased from abcr - Gmbh (Karlsruhe, Germany), were used as received without any purification (Fig. 1). Tetrahydrofuran (THF) was purchased from Fisher Scientific (Waltham MA, USA). Molar mass and phosphorus content of the different monomers are presented in Table 1.

\subsection{General procedure of flax fabrics modification by radiation grafting}

This procedure involves three steps. In the first step, $2 \mathrm{~g}$ of flax fabrics $\left(9 \times 12 \mathrm{~cm}^{2}\right)$ were dipped $1 \mathrm{~min}$ into a phosphorus FR aqueous solution. A fabric/solution weight ratio of $1 / 10$ was fixed for all the dipping assays. The phosphonated monomer concentration in aqueous solution was adjusted to achieve a weight percentage of $1 \%, 5 \%$ and $10 \%$ for hPAAPC 1 , hPMAPC 1 , APA and VPA. For MAPC1 and MVP, a concentration of $10 \mathrm{wt} \%$ was chosen in order to compare with previous studies [15]. Flax fabrics were also dipped in the $98 \mathrm{wt} \%$ MVP as prepared by the supplier in order to highlight the importance of the solvent to incorporate efficiently the FR in the bulk of the fibers. Fabrics were then placed under an extractor hood until total solvent evaporation. In the second step, the dried fabrics were irradiated at radiation doses of 10,20 and $50 \mathrm{kGy}$ in air, at room temperature, using an electron beam accelerator (energy $9.8 \mathrm{MeV}$, power $27 \mathrm{~kW}$ ) realized by Ionisos SA (Chaumesnil, France). In the third step, each irradiated fabrics were washed for $1 \mathrm{~min}$ with

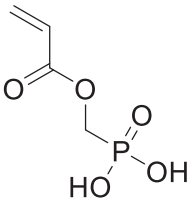

hPAAPC1<smiles>C=CCP(=O)(O)O</smiles>

APA

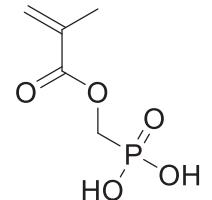

hPMAPC1<smiles>C=CP(=O)(O)O</smiles>

VPA<smiles>C=C(C)C(=O)OCP(=O)(OC)OC</smiles>

MAPC1
Fig. 1. Phosphorus-based molecules used in this study. 
Table 1

Phosphorus content of the monomers used in this study.

\begin{tabular}{ll}
\hline Phosphonate monomers & Phosphorus content (wt\%) \\
\hline hPAAPC1 & 18.7 \\
hPMAPC1 & 17.2 \\
MAPC1 & 14.9 \\
\hline APA & 25.4 \\
VPA & 28.7 \\
MVP & 22.8 \\
\hline
\end{tabular}

water at room temperature to remove the unbounded monomers, oligomers or polymers. Preliminary tests have confirmed that three washing steps are sufficient to remove molecules able to be extracted from flax. Some fabrics were washed as previously described while using THF instead of demineralized water to compare the effect of the solvent washing type on the final phosphorus content. The washed fabrics were then placed under a fume hood until total removal of the residual absorbed solvent.

The Table 2 summarizes the different samples prepared in this study.

\subsection{Characterization}

\subsubsection{X-ray fluorescence elemental analysis}

An "Oxford XMET 5100" X-ray fluorescence analyzer was used to determine the phosphorus elemental content in treated flax fabrics. Samples were fixed on a flat polymer holder which does not contain any phosphorus traces. This holder was used to flatten fabrics to reduce instrumental errors. Analyses were achieved under atmospheric pressure, without any preparation. The following settings were used as default: $13 \mathrm{kV}$ and $45 \mu \mathrm{A}$. Each spectrum was collected with a fixed measuring time (60 s). As the intensity of the phosphorus $\mathrm{K} \alpha$ peak is proportional to its concentration, the data were collected and converted by a simple calculation to determine the weight percentage of phosphorus. This instrument was calibrated before starting analysis using samples of known phosphorus concentration (measured by ICP-AES [15]). Thus, a calibration curve was drawn with a high correlation coefficient (supporting information 1). For each sample, peak intensity was converted into phosphorus weight percentage using this calibration curve. Phosphorus content for each sample was measured 3 times in order to reduce the measurement errors and the error bar was calculated. The highest error of these measurements was $8.7 \%$ for a low phosphorus content which was considered as acceptable value.

\subsubsection{Swelling of elementary flax fibers}

Swelling behavior of flax fibers in water and THF was observed between two glass plates by optical microscopy in transmission mode with a Laborlux 12 Pol S (Leica, Germany) equipped with a mono-CDD Sony digital camera (resolution $1600 \times 1200$ pixels) and an image acquisition software $\operatorname{Archimed}^{\circledR}$ (Microvision Instruments, France). The elementary flax fiber was fixed on the bottom glass plate between two double-sided tapes with around $1 \mathrm{~cm}$ apart. The solvent (THF or water) contained in a pipette was injected by capillarity between the two glass plates. No agitation was applied to the system. The evolution of the fiber's diameter was monitored and measured.

\subsubsection{Scanning electron microscopy SEM}

A scanning electron microscope (FEI Quanta 200) was used to obtain fiber micrographs. The fibers were analyzed and the micrographs were obtained using a vertical sample holder under high vacuum at a voltage of $12.5 \mathrm{kV}$ and a working distance of $10 \mathrm{~mm}$. The SEM is equipped with an energy dispersive X-ray spectroscopy, EDX, (Oxford INCA Energy system) which was used to locate the phosphorus in the flax fibers.

Table 2

Different samples prepared in this study with various concentrations, radiation doses, washing solvents and corresponding phosphorus and flame retardant contents.

\begin{tabular}{|c|c|c|c|c|c|}
\hline FR Monomer & Concentration (wt\%) & Dose (kGy) & Washing solvent & P-content (wt\%) & FR-content (wt\%) \\
\hline \multirow[t]{6}{*}{ VPA } & 1 & 50 & Water & 0,26 & 0,91 \\
\hline & 5 & 10 & Water & 0,59 & 2,06 \\
\hline & & 20 & Water & 0,90 & 3,14 \\
\hline & & 50 & Water & 1,10 & 3,83 \\
\hline & & & THF & 1,94 & 6,76 \\
\hline & 10 & 50 & Water & 1,38 & 4,81 \\
\hline \multirow[t]{7}{*}{ APA } & 1 & 50 & Water & 0,17 & 0,67 \\
\hline & 5 & 10 & Water & 0,15 & 0,59 \\
\hline & & 20 & Water & 0,25 & 0,98 \\
\hline & & 50 & Water & 0,40 & 1,57 \\
\hline & & & THF & 1,09 & 4,29 \\
\hline & & 100 & Water & 0,41 & 1,61 \\
\hline & 10 & 50 & Water & 0,34 & 1,34 \\
\hline \multirow[t]{6}{*}{ hPAAPC1 } & 1 & 50 & Water & 0,21 & 1,12 \\
\hline & 5 & 10 & Water & 0,51 & 2,73 \\
\hline & & 20 & Water & 0,61 & 3,27 \\
\hline & & 50 & Water & 0,51 & 2,73 \\
\hline & & & THF & 1,19 & 6,37 \\
\hline & 10 & 50 & Water & 0,90 & 4,82 \\
\hline \multirow[t]{6}{*}{ hPMAPC1 } & 1 & 50 & Water & 0,20 & 1,16 \\
\hline & 5 & 10 & Water & 0,62 & 3,60 \\
\hline & & 20 & Water & 0,72 & 4,18 \\
\hline & & 50 & Water & 0,58 & 3,37 \\
\hline & & & THF & 1,16 & 6,74 \\
\hline & 10 & 50 & Water & 1,15 & 6,68 \\
\hline \multirow[t]{2}{*}{ MVP } & 10 & 50 & Water & 0,82 & 3,60 \\
\hline & 98 & 50 & Water & 0,62 & 2,72 \\
\hline MAPC1 & 10 & 50 & Water & 1,06 & 7,11 \\
\hline
\end{tabular}




\subsection{4. ${ }^{1} \mathrm{H}$ nuclear magnetic resonance $\left({ }^{1} \mathrm{H}\right.$ NMR $)$}

${ }^{1} \mathrm{H}$ NMR spectra were recorded on Bruker AC 400 instruments, using deuterated water as the solvent and tetramethylsilane as the references for ${ }^{1} \mathrm{H}$ nuclei. Chemical shifts are given in part per million (ppm). The experimental conditions for recording ${ }^{1} \mathrm{H}$ NMR spectra were as follows: flip angle $90^{\circ}$, acquisition time $4.5 \mathrm{~s}$, pulse delay $2 \mathrm{~s}$ and number of scans 16.

\subsubsection{Thermogravimetric analysis (TGA)}

TGA was performed using thermogravimetric analyzer instrument (Setaram Setsys). Samples $(10 \mathrm{mg} \pm 1 \mathrm{mg}$ ) were heated in alumina ceramic crucibles from 30 to $800{ }^{\circ} \mathrm{C}$ at a heating rate of $10^{\circ} \mathrm{C} / \mathrm{min}$ under nitrogen atmosphere $(100 \mathrm{ml} / \mathrm{min})$. Residual weight at $750^{\circ} \mathrm{C}$ (Res750), maximum degradation temperature $\left(\mathrm{T}_{\mathrm{deg}}\right)$ (i.e. temperature of the mass loss rate peak) and maximum peak of mass loss rate (pMLR) were determined.

\subsubsection{Pyrolysis combustion flow calorimetry (PCFC)}

A pyrolysis combustion flow calorimeter (Fire Testing Technology Ltd., UK) was used to investigate the fire behavior of samples at microscale $(2-4 \mathrm{mg})$. Sample pyrolysis was performed at a heating rate of $1{ }^{\circ} \mathrm{C} / \mathrm{s}$ under a nitrogen flow $(100 \mathrm{ml} / \mathrm{min})$ from 90 to $750^{\circ} \mathrm{C}$ (anaerobic pyrolysis - method A according to the standard ASTM D7309). Pyrolysis gases were carried to a combustor in the presence of a $\mathrm{N}_{2} / \mathrm{O}_{2}(80 / 20)$ mixture. In such conditions, all gases are fully oxidized. Heat Rate Release (HRR) is calculated according to Huggett's relation [33] which states that $1 \mathrm{~kg}$ of consumed oxygen corresponds to $13.1 \mathrm{MJ}$ of heat release. Each test on a single sample was performed twice to ensure the reproducibility of our measurements.

The peak of heat rate release (pHRR), the temperature at pHRR $\left(T_{\max }\right)$, the total heat release (THR) and the heat of complete combustion $(\Delta \mathrm{h}$ from the ratio between THR and the fraction of mass loss) were determined.

\subsubsection{Preliminary fire test on fabrics}

An unstandardized fire test was carried out to assess the flammability of some fabrics [15,16]. Fabrics samples (around $0.5 \mathrm{~g}-$ dimensions: $4 \times 2 \mathrm{~cm}^{2}$ ) are pinched between two glass plates and maintained vertically. A cigarette lighter is used to ignite the top of fabrics. This method allows the fabrics self-extinguishment to be evaluated, i.e. absence of flame spreading. The residue is also weighted after complete burning. For self-extinguishing fabric, complete burning is reached by multiple flame applications. As already shown in our previous works $[15,16]$, this test is reproducible and allows assessing the self-extinguishment and the charring of the fabrics. This information is sufficient to guide our research and development efforts.

\subsubsection{Cone calorimetry}

While cone calorimeter is the most common fire bench-scale test, some tests were carried out using a FTT device on untreated flax fabric and fabric modified with $5 \mathrm{wt} \%$ of VPA and irradiated at $50 \mathrm{kGy}$ (phosphorus content $1.1 \mathrm{wt} \%$ ). Even if cone calorimeter is rather used for thermally-thick materials, it can be also valuable for thermally-thin ones [34-37]. These tests were performed to assess the impact of a significant amount of phosphorus on flame retardancy at various heat fluxes $\left(25,35,50\right.$ and $\left.75 \mathrm{~kW} / \mathrm{m}^{2}\right)$. The distance between the radiant cone and the sample is $25 \mathrm{~mm}$. The $10 \times 10 \mathrm{~cm}^{2}$ fabrics are positioned horizontally on a sample holder and are wrapped in aluminum foil. The bottom surface is insulated by rock wool. A metal grid is placed on the upper surface of the fabric to avoid deformation of the fabric during the test as suggested by Tata et al. [38]. Air flow is fixed at $24 \mathrm{~L} / \mathrm{s}$. The heat release rate (HRR) is calculated according to the Huggett's relation as in
PCFC [33]. Two tests were performed for each fabric and each condition.

\section{Results and discussion}

\subsection{Grafting of phosphonated FRs}

\subsubsection{Influence of the FR solution concentration}

Fabrics were dipped in different FR solutions with different concentrations then irradiated at different radiation doses.

As already shown in previous works [16,17], the impregnation step allows to control the final FR-content in flax fabrics. The monomers used in this study penetrated in all cases to the bulk of the fibers according to SEM-EDX observations (supporting information 2). Fig. 2 shows a linear correlation between the weight gain and the FR concentration in solution. Fibers weight after impregnation step could increase from $2.0 \pm 0.5 \mathrm{wt} \%$ for a $1 \mathrm{wt}$ $\%$ FR solution to $19 \pm 2 \mathrm{wt} \%$ for a $10 \mathrm{wt} \%$ FR solution. The difference in weight gain among the different FRs is quite limited. However, MVP exhibits a different behavior in comparison to other molecules. In fact, in impregnation step, water is used as a solvent. A long time is needed for complete solvent removal. As a result, MVP seems to be quite volatile and more easily removed from flax fabrics than other monomers.

After radiation grafting at $50 \mathrm{kGy}$ and washing with water, phosphorus (P) content was measured by X-ray fluorescence. Then P-content was converted into FR-content by a simple calculation (Fig. 3). For $1 \mathrm{wt} \%$ of FR concentration in solution, there was no significant difference in FR grafting on flax fabrics. The phosphorus content and the grafted amount of FRs of the resulting fabrics were respectively around $0.2 \mathrm{wt} \%$ and $1.0 \mathrm{wt} \%$. The difference is more significant for higher concentrations. As shown in Fig. 3, hPMAPC1 and MAPC1 present the highest grafted concentration with no significant difference between them (6.7 and $7.1 \mathrm{wt} \%$ respectively) when FR concentration in solution was fixed at $10 \mathrm{wt} \%$. This is certainly related to the location of the phosphonate function in the molecule. As it is far from the $\mathrm{C}=\mathrm{C}$ double bond, it has not a significant influence on the reactivity of this double bond. However, a clear difference was observed between VPA and its phosphonate MVP. At a $10 \mathrm{wt} \%$ of FR in aqueous solution, $4.8 \mathrm{wt} \%$ of VPA and $3.6 \mathrm{wt} \%$ of MVP were grafted on flax fabrics. This difference could not be correlated to the reactivity of these monomers but rather to

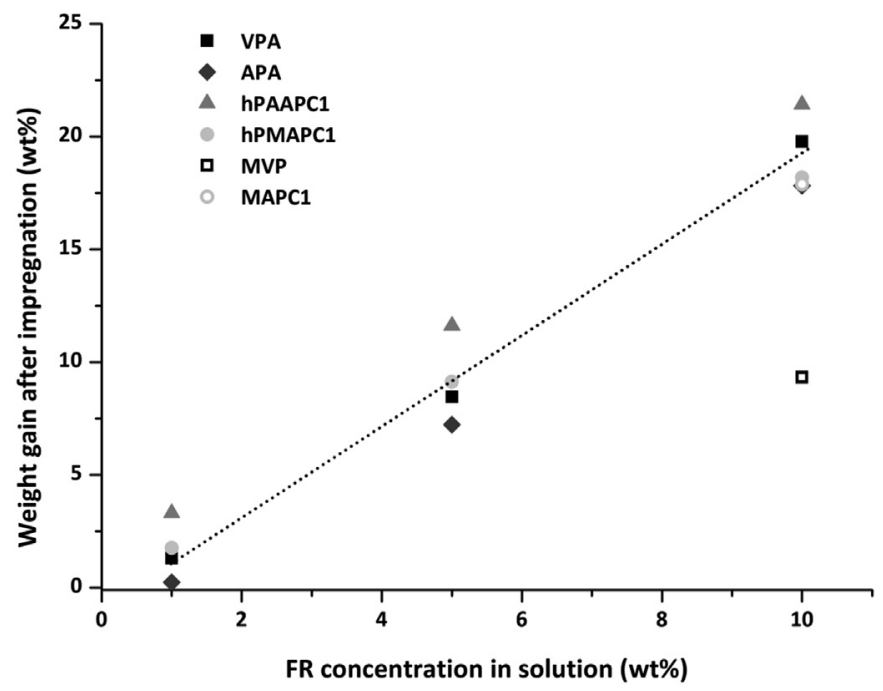

Fig. 2. Weight gain after impregnation versus FR concentration in solution (wt\%). 


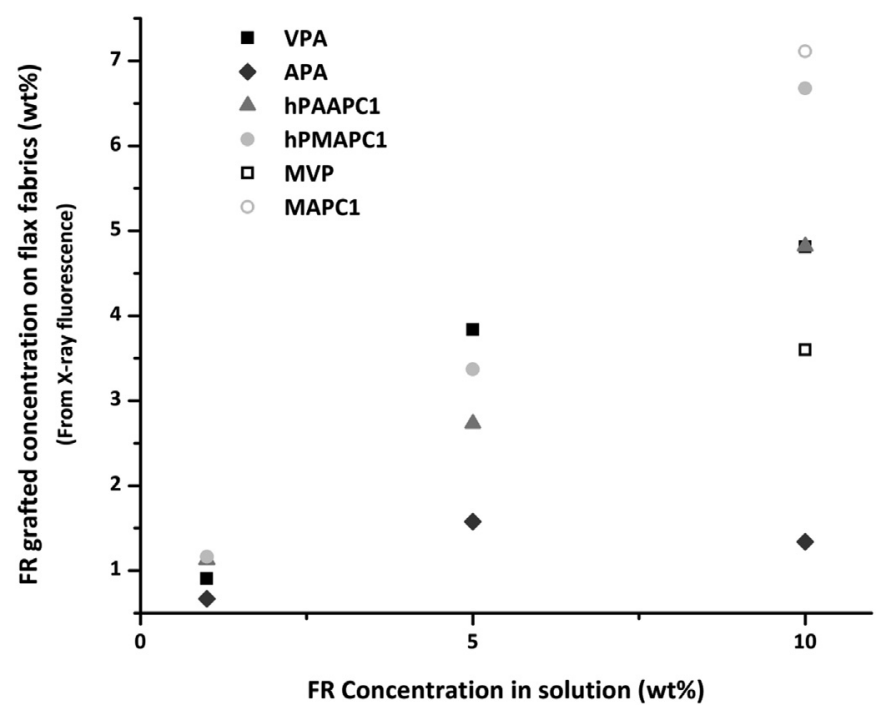

Fig. 3. FR grafted concentration on flax fabrics (from X-ray fluorescence) at different concentrations in aqueous solution irradiated at a dose of $50 \mathrm{kGy}$ and washed by water.

the weight gain after impregnation. As the Fig. 2 shows, when flax fabric was dipped in a $10 \mathrm{wt} \%$ VPA solution, the weight gain was around $19.8 \mathrm{wt} \%$, whereas it was $9.3 \mathrm{wt} \%$ with MVP at the same concentration. The fabrics were dried in both cases, and weight gain was followed over few days to make sure of its stability after drying. MVP seems to be more volatile and thus the amount incorporated in dipping step does not remain trapped completely in flax.

A clear difference in the grafting yield was also observed between hPMAPC1 and hPAAPC1. Acrylates are known to be more reactive than methacrylates in radical polymerization [39]. Nevertheless, in this study, grafting yield is higher for hPMAPC1 (see below for a possible explanation). VPA was more easily grafted than APA on flax fabrics. Vinylic monomers are known to be more reactive than allylic monomers [40]. David and Negrell-Guirao [40] noted than under free-radical conditions, the homopolymerization of allyl monomers is unlikely, but if it occurs, the polymerization rate would be very low. Thus, a higher concentration of VPA in the treated flax fabrics was observed.

Flax fabrics were also dipped in the $98 \mathrm{wt} \%$ MVP as prepared by the supplier. Weight gain after impregnation and FR grafting content were equal to $99.6 \mathrm{wt} \%$ and $2.7 \mathrm{wt} \%$ respectively. The EDX observations showed that phosphorus penetrated to the bulk of the fiber in all cases (Supporting information 2). However, phosphorus content obtained from X-ray fluorescence showed that flax impregnated in a $10 \mathrm{wt} \%$ aqueous solution of MVP contained higher phosphorus amount than that impregnated in $98 \mathrm{wt} \%$ MVP solution despite the high weight gain after impregnation. This shows the importance of using the solvent as a way to incorporate effectively and more easily the monomer into the fiber. So, the solvent helps the monomers to diffuse into the bulk of the fiber with a good distribution to promote the grafting on flax components.

\subsubsection{Influence of the radiation dose}

The influence of the irradiation dose is also of first importance. The dose should be high enough to ensure high phosphorus grafting but as low as possible to avoid flax degradation. The phosphonic acids used in this study were grafted at a concentration of $5 \mathrm{wt} \%$ of the FR monomer at different radiation doses. The Fig. 4 shows that acrylic and methacrylic functions grafting are independent of the radiation dose in the studied dose range. A dose of

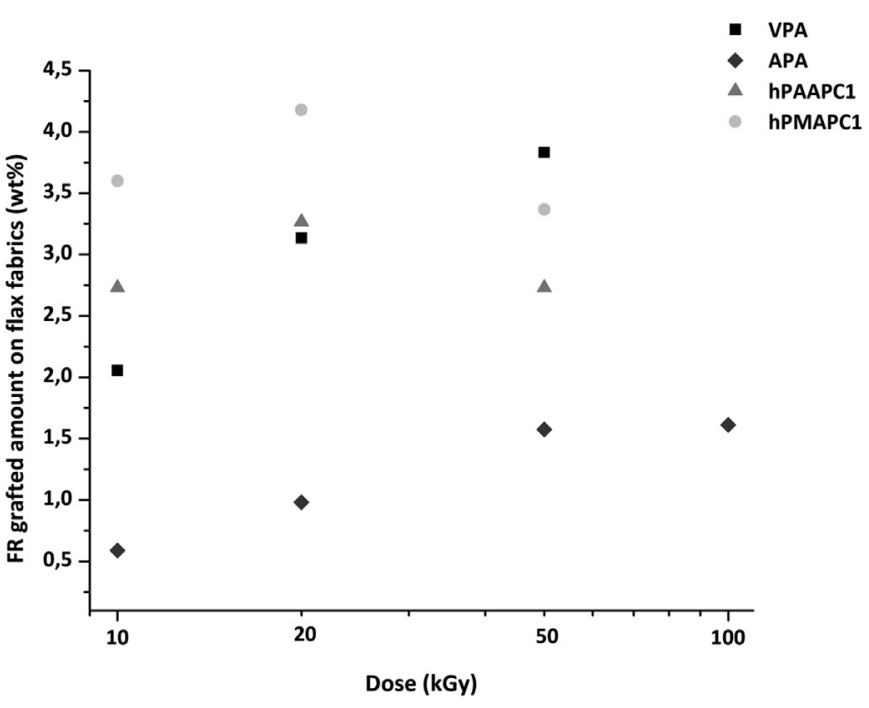

Fig. 4. Influence of radiation dose on grafted FR amount for flax fabrics impregnated with a solution containing $5 \mathrm{wt} \%$ of the FR monomer, irradiated and washed with water.

$20 \mathrm{kGy}$ is sufficient to graft enough FRs on flax fabrics without leading to fibers degradation. Vinyl phosphonic acid showed an increase in FR grafting when the radiation dose increases. Allylic function shows a slight increase in monomer grafting between 10 and $50 \mathrm{kGy}$ but the FR content remains very limited. A higher radiation dose was used to observe if the radiation dose range was insufficient to break the stability of the allylic function. The result showed that after $50 \mathrm{kGy}$ (i.e. for $100 \mathrm{kGy}$ ) there is no change in the final phosphorus content in flax fibers. This means that the reactivity of the allylic functions is very low in comparison to the other studied functions.

\subsubsection{Effect of the washing solvent}

Washing step was also studied to understand how to control the final phosphorus content. Water was used as green solvent and THF was used for comparison purpose with previous works [15]. Preliminary test showed the ability of each studied monomer to be dissolved in both solvents. In all cases, water showed to be more efficient than THF in extracting the ungrafted phosphonic molecules (Fig. 5).

To understand this behavior, the evolution of the diameter of flax elementary fibers in solvents was observed by optical microscopy (Fig. 6). The optical observations showed an increase of around $21 \%$ in fiber's diameter when dipped in water whereas there was no change when dipped in THF. These results may explain the effect of the washing solvent on the final phosphorus content in flax fibers. Two phenomena can occur during e-beam irradiation (see Scheme 1).

The e-beam irradiation may involve degradation of some covalent bonds of the structures of the main flax components (cellulose and hemicelluloses) creating free radicals which can initiate the polymerization of the FR monomer (flax initiation). In this case the polymer chains bearing phosphonated groups will be covalently grafted to the elementary fibers structure. In the second case, initiation can be due to the decomposition of monomers units which create free radicals. The polymerization initiated by these radicals (monomer self-initiation) inside the fiber's bulk will result in the formation of free polymer chains. In brief, some polymer chains are covalently grafted to flax (flax initiation), others form ungrafted polymer chains (monomer self-initiation) and others remained without reacting. Thus, when the modified fabrics are 


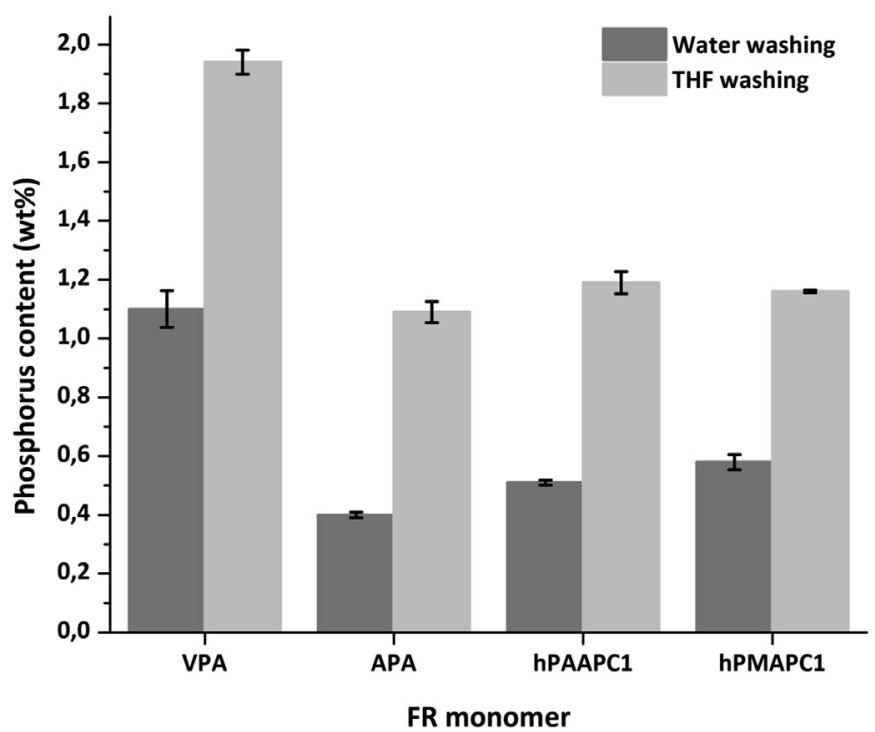

Fig. 5. Influence of the choice of the washing solvent on final phosphorus content (monomers concentration in solution $=5 \mathrm{wt} \%$; radiation dose $=50 \mathrm{kGy}$ ).

dipped in water, the fibers swell, hence opening the pores and increasing the accessibility to the bulk. This gives the possibility for water to extract the unreacted monomers and the ungrafted polymer chains from the bulk of the fibers. Since no swelling occurs in THF, the accessibility to unreacted monomers and ungrafted polymers is more restricted and removal is probably limited to smaller macromolecules.

\subsubsection{Reactivity of the monomers under e-beam radiation}

To understand the effect of the e-beam radiation on the monomers, hPMAPC1, hPAAPC1, MAPC1, VPA, APA and MVP were irradiated at $50 \mathrm{kGy}$ and analyzed by ${ }^{1} \mathrm{H}$ NMR. However for hPAAPC1 and APA the method was not suitable. Indeed, the solid product obtained after irradiation of hPAAPC1 formed a viscous gel when we tried to dissolve it in deuterated water and could not be analyzed by ${ }^{1} \mathrm{H}$ NMR. APA is in solid state at room temperature contrarily to the other monomers used in this study which are in liquid state. Thus, for pure APA no change was observed after irradiation (according to ${ }^{1} \mathrm{H}$ NMR spectrum) which could be due to the low mobility of molecules in solid state. In order to overcome these issues, hPAAPC1 and APA were dissolved in water (10 wt\%) and irradiated at $50 \mathrm{kGy}$. Water was then removed by lyophilization and the obtained sample was analyzed by ${ }^{1} \mathrm{H}$ NMR analysis. ${ }^{1} \mathrm{H}$ NMR spectra (Figs. 7 and 8) show that hPMAPC1 and hPAAPC1 have a greater tendency to polymerize under e-beam irradiation than VPA, MAPC1, MVP and APA. Indeed hPAAPC1 easily polymerizes while the characteristic peaks of this monomer no longer appear on the NMR spectrum after irradiation. Only peaks corresponding to the polymer are present evidencing that the conversion ratio is $100 \%$. hPMAPC1 shows also high rate of polymerization with large signals between 0.6 and $2.7 \mathrm{ppm}$ and between 3.9 and $4.4 \mathrm{ppm}$ corresponding to hydrogens of the polymer chains. Characteristic peaks of monomer are still present between 5.6 and $6.1 \mathrm{ppm}$ corresponding to hydrogen atoms of the carbon-carbon double bond. A comparison of intensities of monomer and polymer peaks evidences a conversion ratio for hPMAPC1 around 99.6\%. However, MAPC1 shows a lower rate of polymerization than hPMAPC1 with small signals between 0.6 and $2.5 \mathrm{ppm}$ and between 3.9 and $4.6 \mathrm{ppm}$ corresponding to hydrogens of the polymer chains. Characteristic peaks of monomer are still present between 5.7 and $6.2 \mathrm{ppm}$ corresponding to hydrogen atoms of the carbon-carbon double bond. A comparison of intensities of monomer and polymer peaks evidences a conversion ratio for MAPC1 around $61.5 \%$. Irradiated VPA spectrum showed a good conversion rate with
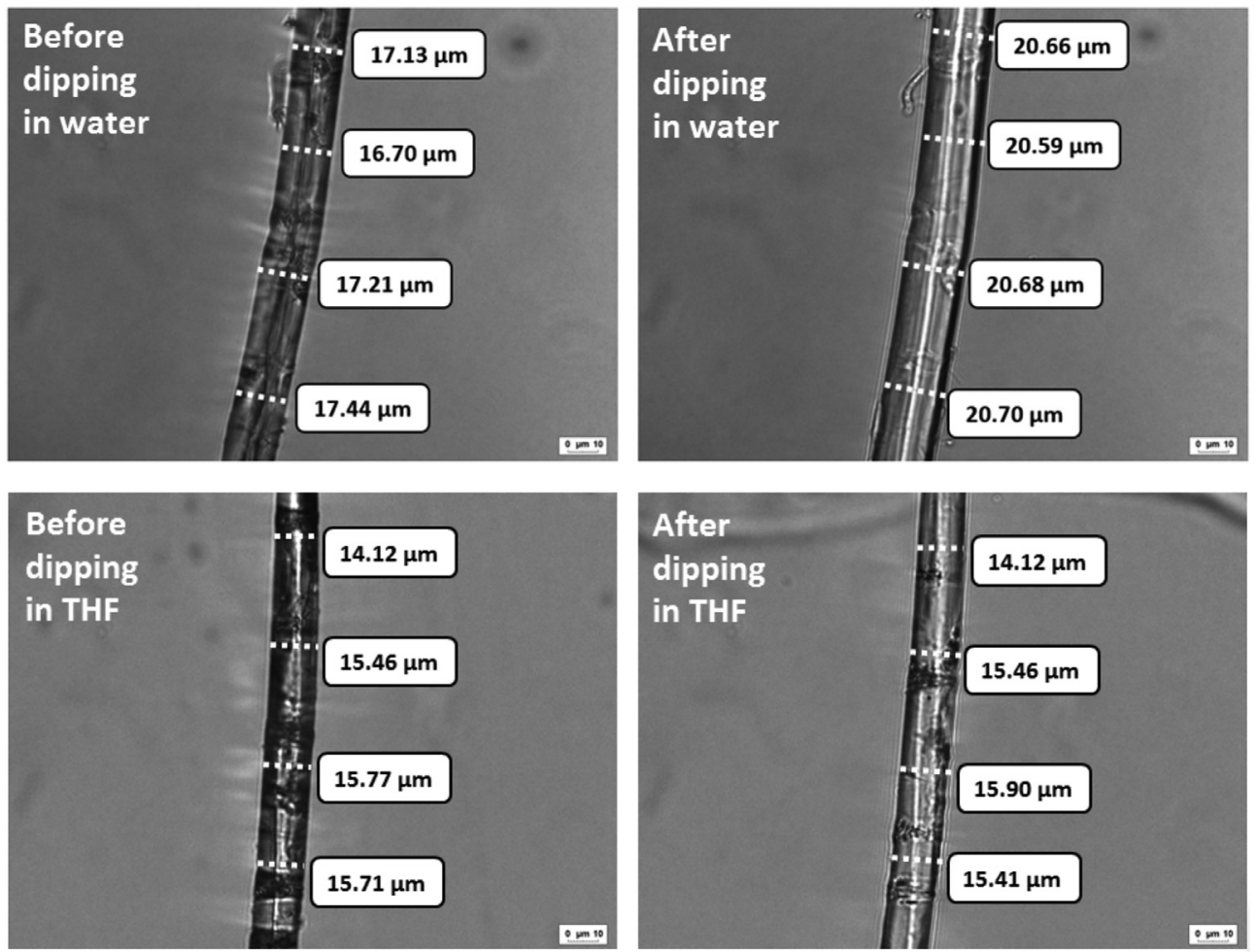

Fig. 6. Influence of water and THF on the diameter of flax elementary fibers. 


\section{Monomer self-initiation (homopolymerization)}

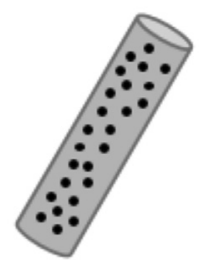

\section{e-beam radiation}

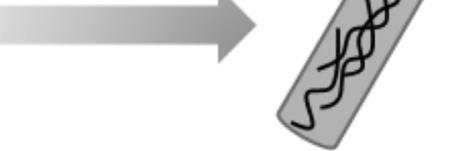

\section{Flax initiation (Grafting)}
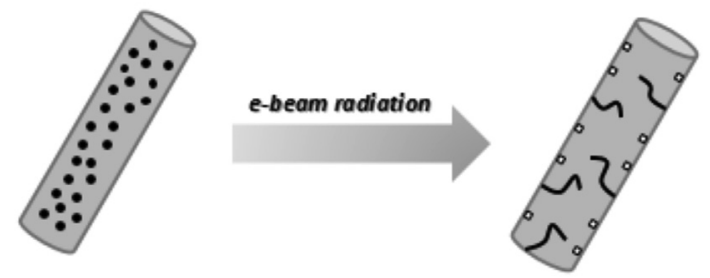

- Unreacted Monomers

- Grafted Monomers

( Grafted Oligomers / Polymers

$\sim$ Ungrafted Polymers

Scheme 1. Monomers interactions with e-beam radiation.

polymer characteristic peaks between 0.9 and $2.6 \mathrm{ppm}\left(\mathrm{CH}_{2}\right.$ and $\mathrm{CH})$. The comparison of intensities of the peak centered at $6 \mathrm{ppm}$ characteristic of the monomer (hydrogens of the vinyl bond) and signals between 0.9 and $2.6 \mathrm{ppm}$ corresponding to the formed polymer gives a conversion rate about $82.5 \%$. APA and MVP are the monomers exhibiting the lowest ability to polymerize under irradiation. The comparison of intensities of APA monomer (signals centered at 5.1 or $5.7 \mathrm{ppm}$ ) and its polymer (signal between 0.8 and $2 \mathrm{ppm}$ ) characteristic peaks gives a conversion rate of about $18.9 \%$. Also, the comparison of intensities of the peak centered at $6.2 \mathrm{ppm}$ characteristic of the MVP monomer (hydrogens of the vinyl bond) and signals between 1 and $2.7 \mathrm{ppm}$ corresponding to the formed polymer gives a conversion rate about $9.1 \%$. These results may provide new insights about the phenomena occurring during mutual radiation grafting. Two phenomena are competing: the first is the self-initiation where the radical is formed on the monomer units under e-beam radiation and leads to the formation of polymer chains unbound to flax components, and the second is the flax initiation where the radicals are formed on flax fibers promoting FR polymer chains bound to flax components. However, it is difficult to confirm which flax component is the most affected by the e-beam irradiation and the grafting, and this point should be further investigated. In fact, a large fraction of hPMAPC1 and hPAAPC1 can homopolymerize easily with self-initiation under e-beam radiation inside the fiber. However, the X-ray fluorescence measurements showed that the final flame retardant content was higher for hPMAPC1 than for hPAAPC1. So in comparison to hPAAPC1, the probability for hPMAPC1 to react with radicals formed from flax structure under irradiation to give more polymer chains initiated from the flax structure is higher. hPAAPC1 would form more free polymer chains due to self-initiation inside fiber's bulk with less covalent bonds with flax components. Even if these free polymers chains could have a high polymerization degree, their extraction during washing step occurs at a certain extent. Therefore, final hPAAPC1 content is lower than hPMAPC1 content. VPA and MAPC1 also homopolymerizes but at moderate rate whereas MVP homopolymerizes at very low rate under e-beam radiation. However, VPA, MAPC1 and MVP content in flax fabrics was quite high. We assume that this is due to a better compromise between the ability to self-initiated homopoplymerization and the high reactivity of

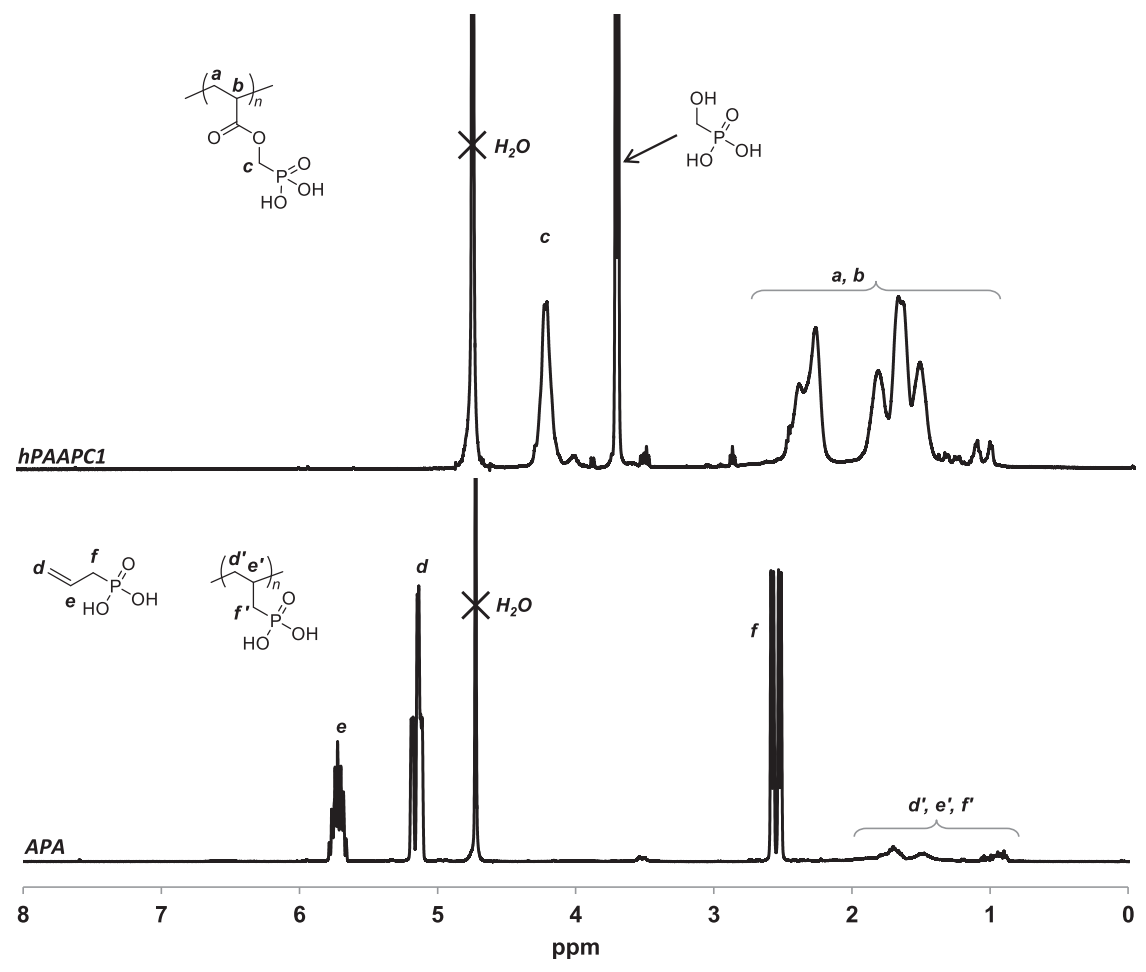

Fig. 7. ${ }^{1} \mathrm{H}$ NMR spectra of hPAAPC1 and APA dissolved in water and irradiated at $50 \mathrm{kGy}$. 


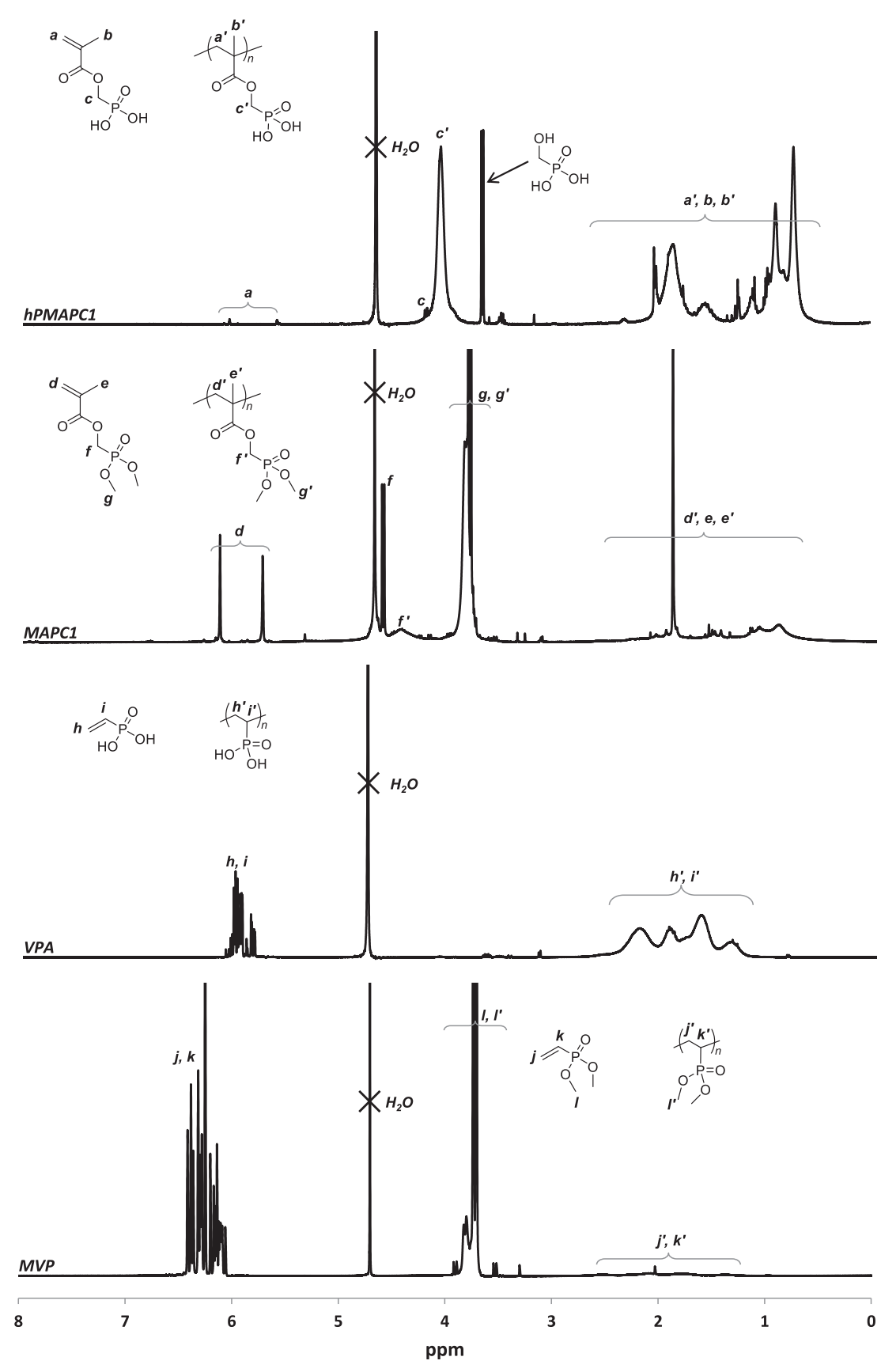

Fig. 8. ${ }^{1} \mathrm{H}$ NMR spectra of hPMAPC1, MAPC1, VPA and MVP irradiated at $50 \mathrm{kGy}$.

these monomers with radicals formed on flax structure leading to the linkage between monomer units or oligomers and flax components. APA has also a low homopolymerization rate when solubilized in water and does not polymerize under irradiation in solid state. Then its reactivity with radicals formed on flax structure under e-beam radiation is believed to be weak leading to a low APA content in the treated flax fabrics.

The phosphorus FRs location was assessed using SEM-EDX. In our previous work [15] MVP and MAPC1 were grafted on flax fabrics. Whereas MVP was grafted into the bulk of the elementary fiber, MAPC1 was located only on the surface. Note that THF was used as solvent during impregnation step. In the present article, water was used as solvent and all molecules penetrated into the bulk (supporting information 2). This confirms that differences in final phosphorus content cannot be assigned to changing ability of molecules to diffuse into flax fibers but are due to differences of monomer reactivity under e-beam radiation.

\subsection{Flame retardancy of modified flax fabrics}

Thermal degradation and flame retardancy of the modified flax fabrics was assessed by TGA, PCFC, cone calorimeter and a simple fire test.

\subsubsection{Thermal degradation at microscale}

The effect of the phosphorus content on the flax fabrics was studied through thermogravimetric analysis and pyrolysis combustion flow calorimetry (anaerobic pyrolysis). Main data can be 
found in supporting information 3. Fig. 9 represents the TGA residues at $750{ }^{\circ} \mathrm{C}$ and the total heat release from PCFC under anaerobic pyrolysis. The figure shows that as the phosphorus content in flax fibers increases, the residue yield increases and the THR value decreases. In fact, these tendencies are very common for phosphoruscontaining FRs used to promote charring of a large range of substrates. This means that when phosphorus content increases, promoting char results in an increase of residues yield in TGA. Thus, less species will be produced in vapor phase during pyrolysis resulting in a decrease in total heat release. Residue at $750^{\circ} \mathrm{C}$ of untreated flax is around $14.7 \mathrm{wt} \%$ and its THR is around $9.3 \mathrm{~kJ} / \mathrm{g}$ while it reaches $40.2 \mathrm{wt} \%$ and $1.4 \mathrm{~kJ} / \mathrm{g}$ respectively after treating flax fabrics with $5 \%$ of hPAAPC1 at $50 \mathrm{kGy}$ and washed by THF (1.19 wt\% $P)$. The flame retardancy also remains significant when water washing is applied. Residue at $750^{\circ} \mathrm{C}$ and THR are respectively $35.9 \mathrm{wt} \%$ and $2.8 \mathrm{~kJ} / \mathrm{g}$ for fabrics treated with $10 \%$ MAPC1 at $50 \mathrm{kGy}$ washed with water $(1.06 \mathrm{wt} \% \mathrm{P})$. No systematic differences in terms of efficiency in flame retardancy were observed between the different FRs at the same phosphorus content.

The heat of complete combustion also changes according to the phosphorus content (Fig. 10). $\Delta \mathrm{h}$ of flax fibers is around $10.4 \mathrm{~kJ} / \mathrm{g}$. It decreases as the phosphorus content increases independently of the phosphonated monomer to reach around $3.6 \mathrm{~kJ} / \mathrm{g}$ when phosphorus content of fiber was $1.94 \mathrm{wt} \%$. This means that the carbon from gases released during pyrolysis of treated flax fibers is already partially oxidized when reaching the combustion chamber. These gases could be inactive species like water vapor, carbon monoxide and carbon dioxide. Also, the intensity of the peak of heat rate release (pHRR) and the temperature at pHRR decreased as phosphorus content increased (Fig. 11). The intensity and the temperature of the pHRR are closely connected respectively to the peak of mass loss rate ( $\mathrm{pMLR}$ ) and the temperature of maximal degradation $\left(\mathrm{T}_{\max }\right)$ in TGA, which show the same tendencies, i.e. a decrease when phosphorus content increases. The pHRR of untreated flax fibers is around $208 \mathrm{~W} / \mathrm{g}$ and its temperature is around $364^{\circ} \mathrm{C}$. It decreased to $39 \mathrm{~W} / \mathrm{g}$ with a temperature of $255^{\circ} \mathrm{C}$ when phosphorus content reached $1.94 \mathrm{wt} \%$. So, in the presence of phosphorus the degradation of flax fibers would start at lower temperatures with a slower rate. This was also confirmed by TGA results where the peak of mass loss rate (pMLR) decreased from 19.3\%/min to $9 \% /$ min and the temperature of maximal degradation decreased from $345^{\circ} \mathrm{C}$ to $268^{\circ} \mathrm{C}$ for untreated flax and flax containing $1.94 \mathrm{wt} \% \mathrm{P}$ respectively.

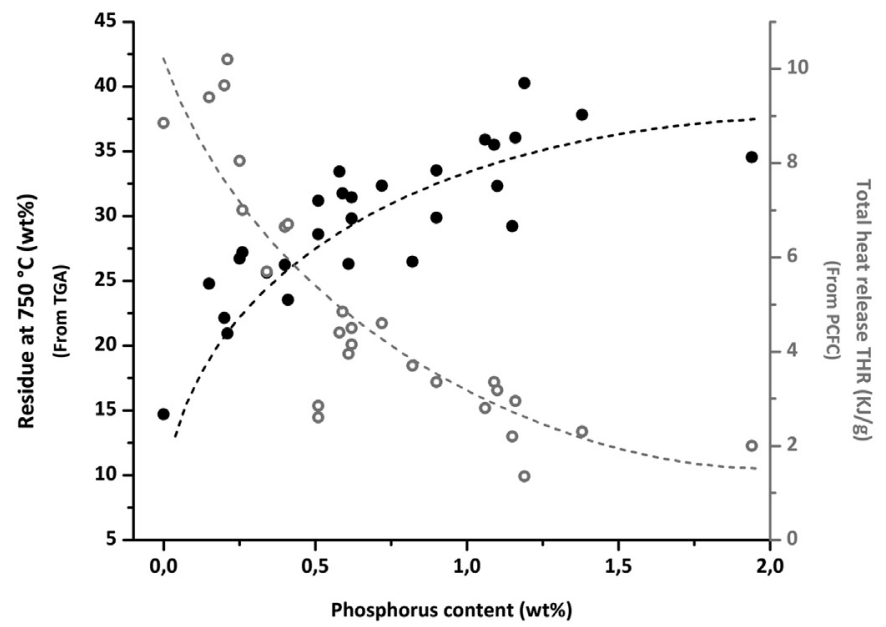

Fig. 9. Influence of phosphorus content on residue content using TGA and total heat release using PCFC (dotted lines are only guidelines for eyes).

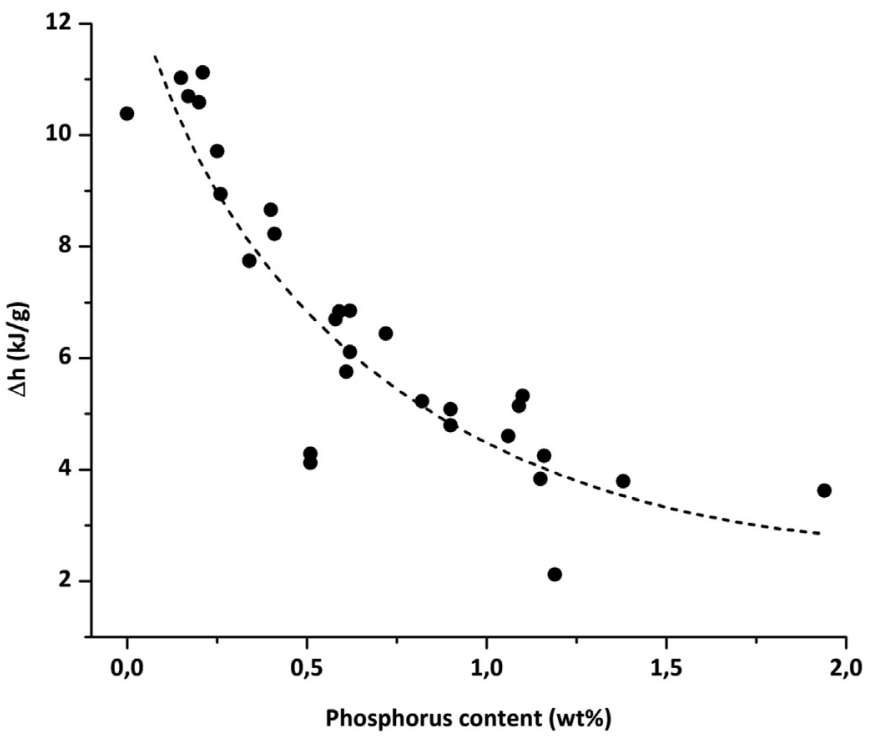

Fig. 10. Influence of phosphorus content on the heat of complete combustion $(\Delta \mathrm{h})$ of flax fibers (dotted lines are only guidelines for eyes).

\subsubsection{Effect of phosphorus on the flammability of flax fabrics}

Fire tests were performed on flax fabrics modified by the phosphonated FRs in order to assess the effect of this modification on flame retardancy (data are reported in supporting information 4). Flax fabrics usually form a low residue amount after ignition which is next degraded by thermo-oxidation leaving a char yield close to $0 \%$. Three different fire behaviors were observed in this study. The first one is the flame propagation with the formation of a stable residue which does not degrade by thermo-oxidation. It is observed even at low P-content. The second one is self-extinguishing where the flame propagation is inhibited (at higher P-content). The third one is non-flammable behavior where fabrics do not ignite and no flame is observed (highest P- content). In the second and third behaviors, multiple ignitions using a cigarette lighter are needed to burn the whole sample and to weight the final residue.

Fig. 12 shows the relation between the flame test results and the phosphorus content. It is well known that flame retardancy level depends mainly on the phosphorus content in the sample [41]. Selfextinguishing behavior was observed when phosphorus concentrations in flax fabrics exceeded $0.5 \mathrm{wt} \%$. Non-flammable behavior was observed when phosphorus content was higher than $1.2 \mathrm{wt} \%$. Moreover, char yield is observed to increase monotonously with phosphorus content.

VPA, hPAAPC1 and hPMAPC1 do not allow obtaining selfextinguishing fabrics when grafting was carried out using a FR concentration of $1 \mathrm{wt} \%$ in aqueous solution and irradiation at $50 \mathrm{kGy}$. APA at any concentration and radiation dose followed by water washing leads to flax fabrics with a low P-content. Thus, the resulting fabrics are flammable with the formation of a stable char. Char yield was low in this case (between 6 and $12 \mathrm{wt} \%$ ).

On the contrary, self-extinguishing fabrics were observed when fabrics were modified with VPA, hPAAPC1 or hPMAPC1 at a concentration of $5 \mathrm{wt} \%$ in aqueous solution with the different irradiation doses (10, 20 and $50 \mathrm{kGy}$ ) and washed by water. Also, the fabrics modified with MVP and MAPC1 with a concentration of $10 \mathrm{wt} \%$ in aqueous solutions and irradiated at $50 \mathrm{kGy}$ followed by water washing shows a self-extinguishing behavior. Char yield in these cases is higher (between 17 and $23.5 \mathrm{wt} \%$ ).

Non-flammable behavior was observed in two cases. The first one concerns the fabrics modified with VPA, hPAAPC1 and hPMAPC1 at a $10 \mathrm{wt} \%$ concentration in aqueous solution and 

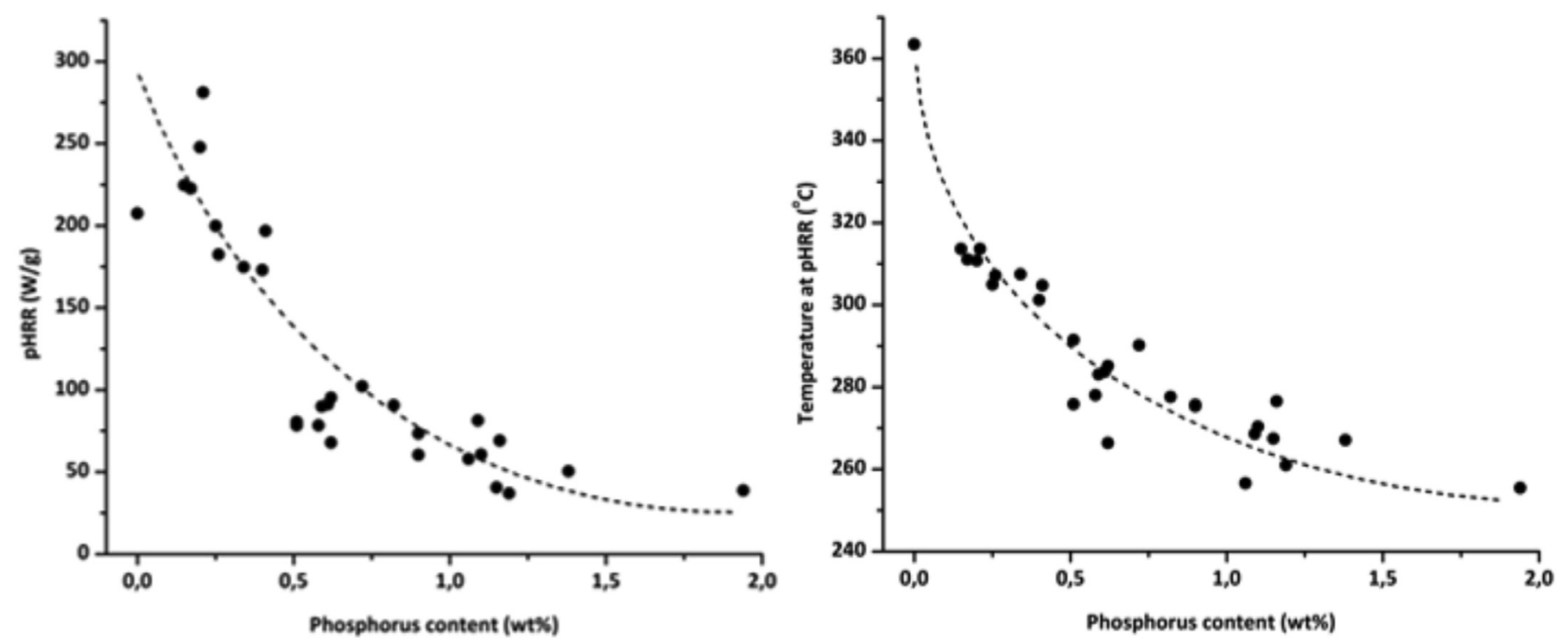

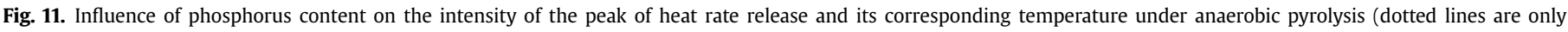
guidelines for eyes).

irradiation dose of $50 \mathrm{kGy}$ followed by water washing. The second case corresponds to fabrics modified with VPA, hPAAPC1, hPAAPC1 and APA at lower concentration of $5 \mathrm{wt} \%$ and the same irradiation dose of $50 \mathrm{kGy}$ but washed with THF. It is important to note that this was the only case where the modification with APA showed a good flame retardancy behavior. In all these cases, this nonflammable behavior was observed because of the high phosphorus content in these fabrics. Note that char yield was systematically between 24 and $31 \mathrm{wt} \%$.

No specific influence of FR structure on flammability was noted. In other words, flammability depends only on phosphorus content while phosphorus content depends on a couple of parameters, i.e. FR concentration in solution, radiation dose, FR reactivity upon irradiation and washing solvent.

Fig. 13 shows the correlation curve between the results obtained by PCFC and the preliminary fire test for the samples tested in this work and in a previous study [16]. Note that experimental error on

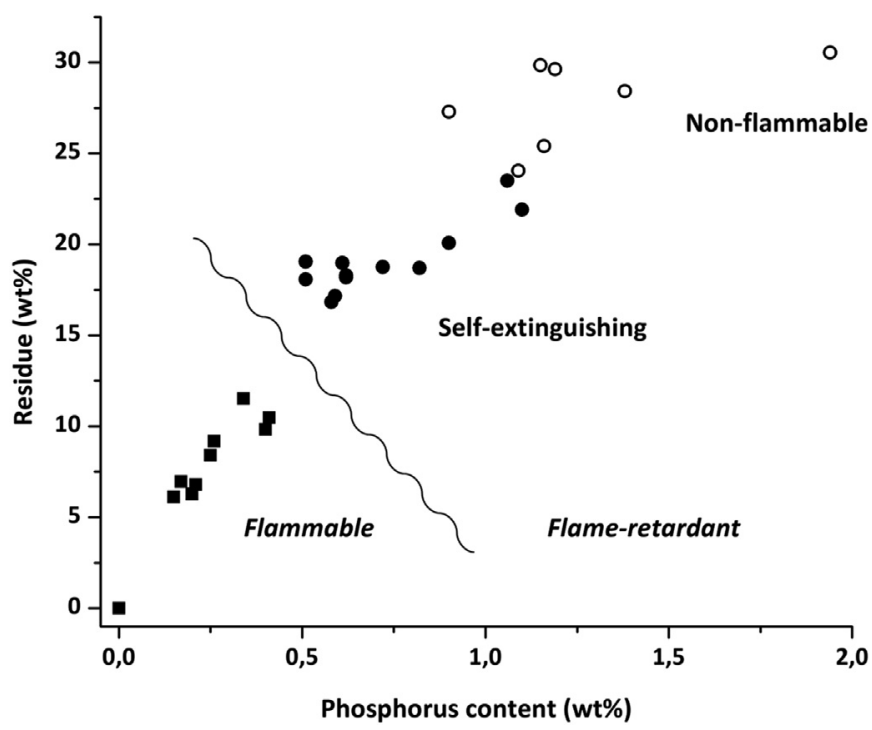

Fig. 12. Char yield versus phosphorus content obtained from fire test.
THR is estimated to $1 \mathrm{~kJ} / \mathrm{g}$. This curve makes it possible to evaluate the flammability of flax fabrics modified by phosphonated molecules from the coordinates of the point on the curve. Fig. 13 shows that when the phosphorus level is greater than $0.5 \mathrm{wt} \%$, total heat release is lower than $5 \mathrm{~kJ} / \mathrm{g}$ and flame retarded flax fabrics (i.e. selfextinguishing fabrics according to the preliminary fire test) are obtained.

\subsubsection{Flammability of flax fabrics under forced flaming conditions}

Heat release rate curves obtained from cone calorimeter tests at $35 \mathrm{~kW} / \mathrm{m}^{2}$ are shown in Fig. 14. Main flammability data are listed in Table 3. All HRR curves have the same shape corresponding to thermally thin materials [42].

Time to ignition (TTI) decreases after the modification of flax fabrics with VPA. TTI of unmodified flax fabric at $35 \mathrm{~kW} / \mathrm{m}^{2}$ is $27 \mathrm{~s}$. After radiation grafting of VPA, the time to ignition decreases to $12 \mathrm{~s}$. This is in agreement with results obtained at microscale. Indeed, the

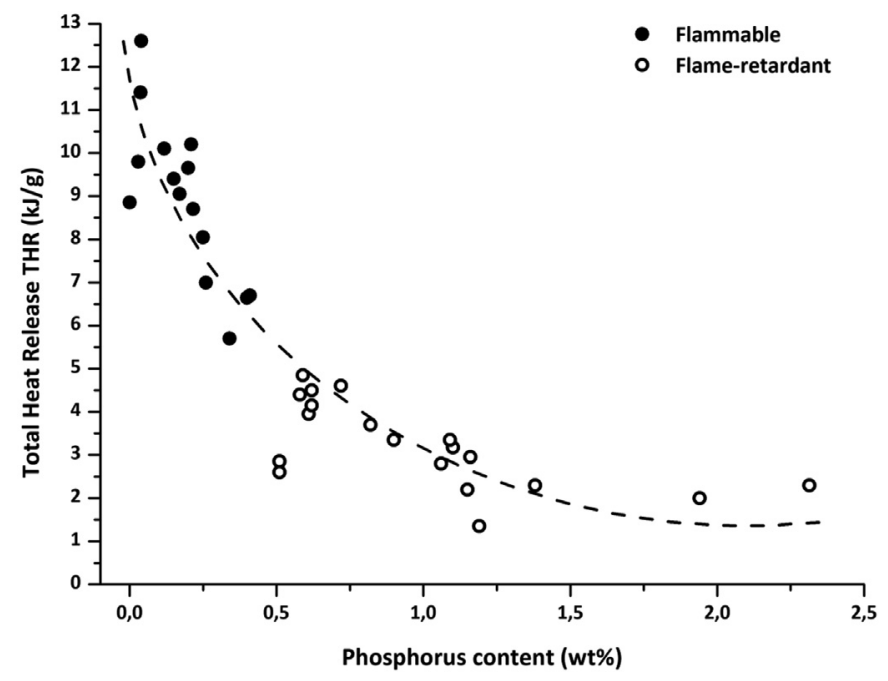

Fig. 13. Total heat release (PCFC) and flammability of flax fabrics in function of phosphorus content (dotted line is only guideline for eyes). 


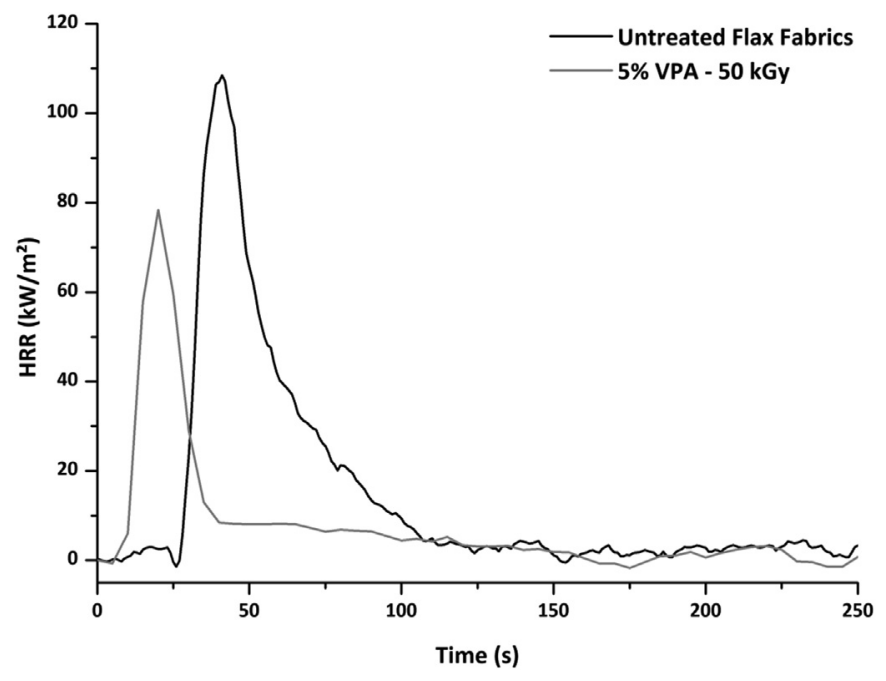

Fig. 14. HRR curves at heat flux of $35 \mathrm{~kW} / \mathrm{m}^{2}$ for untreated flax fabrics and for modified fabrics with a solution of $5 \mathrm{wt} \%$ of VPA and irradiated at $50 \mathrm{kGy}$.

maximum degradation temperature in PCFC decreased from $364^{\circ} \mathrm{C}$ to $272{ }^{\circ} \mathrm{C}$ after modification with $5 \mathrm{wt} \%$ of VPA at $50 \mathrm{kGy}$. Thus, the modification of the flax fabric by VPA reduces its thermal stability and the flax fabric reaches ignition at lower temperatures. Note that the decrease in TTI becomes less significant, after modification of flax, at high heat flux. At $25 \mathrm{~kW} / \mathrm{m}^{2}$, the TTI of unmodified flax is $78 \mathrm{~s}$ and that of flax modified by radiation grafting is $38 \mathrm{~s}$, while at $75 \mathrm{~kW} / \mathrm{m}^{2}$ the TTI becomes similar in both cases at $3 \mathrm{~s}$.

For thermally thin materials, TTI depends on heat flux according to the equation (1) [42]:

$T T I=l \rho c \frac{T_{i g}-T_{0}}{q_{e x t}^{\prime \prime}-C H F}$

with $l$ the thickness, $c$ the specific heat, $\rho$ the density, $T_{i g}$ the temperature at ignition, $T_{0}$ the room temperature, $q_{\text {ext }}^{\prime \prime}$ is the external heat flux and CHF the critical heat flux. The CHF is the minimal heat flow required to ignite the sample. It can be determined by extrapolating the curve $1 /$ TTI versus heat flux for $1 / \mathrm{TTI}=0$. For unmodified flax, the calculated critical heat flow is $26.8 \mathrm{~kW} / \mathrm{m}^{2}$ while after modification, this value decreases to $22.5 \mathrm{~kW} / \mathrm{m}^{2}$. Therefore, CHF seems to be slightly lower after VPA grafting, probably because of the decrease in thermal stability. It should be noted that those values are calculated and may differ from the experimental results. For example, the CHF of unmodified fabric is $26.8 \mathrm{~kW} / \mathrm{m}^{2}$, but the unmodified fabric ignites at $25 \mathrm{~kW} / \mathrm{m}^{2}$ even if its calculated $\mathrm{CHF}$ is higher.

Peak of heat release rate (pHRR) increases obviously when heat flux increases. However, for each heat flux, pHRR is lower after modification. Its value is $87-180 \mathrm{~kW} / \mathrm{m}^{2}$ for unmodified fabric and $61-130 \mathrm{~kW} / \mathrm{m}^{2}$ for the modified one depending on the external heat flux. Moreover, the total heat release also decreases by about $50 \%$ at all heat flux after modification. At $35 \mathrm{~kW} / \mathrm{m}^{2}$, it decreases from $10.9 \mathrm{~kJ} / \mathrm{g}$ to $5.4 \mathrm{~kJ} / \mathrm{g}$. This decrease in the total heat release is associated with the increase in the residue content and the decrease in effective heat of combustion (Table 3 ).

The residue content is plotted versus heat flux in Fig. 15. At $25 \mathrm{~kW} / \mathrm{m}^{2}$, the percentage of residue increases from $13 \mathrm{wt} \%$ to $41 \mathrm{wt} \%$ while at $75 \mathrm{~kW} / \mathrm{m}^{2}$, it increases from $2 \mathrm{wt} \%$ to $23 \mathrm{wt} \%$ after modification with VPA. In fact, we observed that unmodified flax fabric produces only ash after burning at $75 \mathrm{~kW} / \mathrm{m}^{2}$, while modified flax fabrics produces char even at high heat flux. However, the stability of the char remains limited, as the percentage of residue decreases with heat flux due to thermo-oxidation at the end of test.

Effective heat of combustion is also significantly reduced after modification. Indeed, as already shown at microscale, phosphorus FR promotes the formation of a carbon-rich char. This char stores a high amount of energy. Consequently the gases released during pyrolysis contain a lower fraction of carbon and the effective heat of combustion is reduced. It can be noted that the effective heat of combustion increases when heat flux increases because of the amount of char decreases.

\section{Conclusion}

In order to prepare flame retarded flax fabrics, 6 different phosphorous monomers were tested. Mutual irradiation was

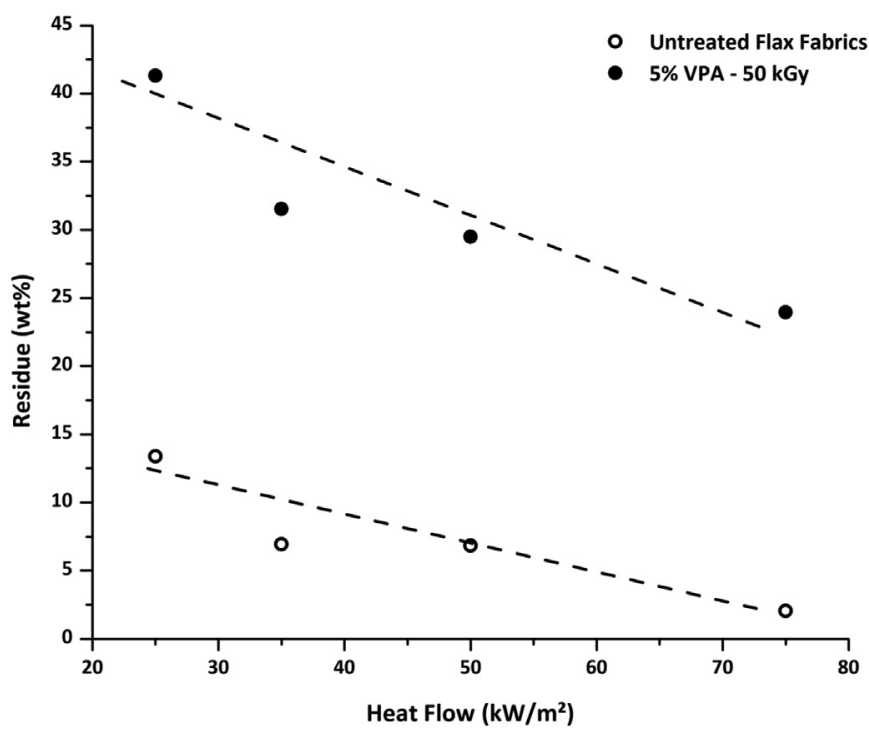

Fig. 15. Evolution of the percentage of residue as a function of the heat flux (The dotted lines are only indications for the eyes).

Table 3

Main data from cone calorimeter tests for untreated and VPA-treated flax fabrics (low and high values have been added between brackets).

\begin{tabular}{|c|c|c|c|c|c|c|}
\hline & Heat flux $\left(\mathrm{kW} / \mathrm{m}^{2}\right)$ & TTI (s) & $\mathrm{pHRR}\left(\mathrm{kW} / \mathrm{m}^{2}\right)$ & THR (kJ/g) & Residue (wt\%) & $\mathrm{EHC}(\mathrm{kJ} / \mathrm{g})$ \\
\hline \multirow[t]{4}{*}{ Untreated flax fabric } & 25 & $78(73-83)$ & $87(84-91)$ & $9.2(8.4-9.9)$ & $13(9-18)$ & $10.5(10.3-10.9)$ \\
\hline & 35 & $27(25-28)$ & $100(92-109)$ & $10.9(10.4-11.3)$ & $7(5.2-8.6)$ & $11.7(11.4-12)$ \\
\hline & 50 & $11(10-11)$ & $127(119-134)$ & $11.6(11.4-11.8)$ & $6.8(4.7-9)$ & $12.4(12.3-12.5)$ \\
\hline & 75 & $3(3-4)$ & $180(177-183)$ & $12.6(12.3-13)$ & $2(0-4.1)$ & $12.9(12.3-13)$ \\
\hline \multirow[t]{4}{*}{ VPA-treated flax fabric (P content $1.1 \mathrm{wt} \%$ ) } & 25 & $38(37-39)$ & $61(58-64)$ & $4.1(4.1-4.1)$ & $41.3(36.4-46.2)$ & $7(6.4-7.6)$ \\
\hline & 35 & $12(13-11)$ & $80(78-81)$ & $5.4(4.7-6.0)$ & $31.5(25-38.1)$ & $7.8(7.6-8)$ \\
\hline & 50 & $8(7-8)$ & $111(108-113)$ & $6.3(5.8-6.7)$ & $29.5(28.5-30.4)$ & $8.9(8.4-9.4)$ \\
\hline & 75 & $3(3-3)$ & $130(128-133)$ & $6.6(6.4-6.8)$ & $24(23-25)$ & $8.7(8.3-9)$ \\
\hline
\end{tabular}


applied to promote the grafting of these monomers on flax. Several phenomena occur simultaneously leading to 1/ FR monomers covalently grafted to flax with or without polymerization, 2/ FR polymerized without grafting to flax, leading to macromolecules trapped into fibers bulk, 3/ unreacted FR monomer.

Monomers containing methacrylic double bond function showed to be the most efficient under e-beam radiation. Allyl phosphonic acid was much less reactive even at high doses of irradiation. Allyl function showed to be not suitable for radiation grafting as it hardly polymerizes and reacts with flax radicals during irradiation step. Acrylic and methacrylic monomers have high tendencies to homopolymerize inside the fiber's bulk. Vinyl phosphonic acid showed to be a well reactive FR in described conditions. It is mostly grafted by reacting with flax radicals during irradiation step. Phosphorus content of VPA is very high which leads to higher phosphorus amounts in the final treated flax fabrics than those treated with other monomers at the same concentration.

Radiation grafting confirms once again to be suitable for lowlignin natural fibers as flax.

The choice of the good solvent in impregnation and washing steps is another key to control the final phosphorus content. Water washing showed to be more effective than THF to remove ungrafted macromolecules and unreacted monomer units. THF seems to extract the ungrafted smallest macromolecules, leaving the biggest ones trapped inside the fiber's bulk. This difference can be ascribed to the capacity of water to swell the flax elementary fibers.

This study also confirms that thermal stability, char residue and flame-retardancy behavior are mainly dependent on phosphorus content and further independent on the monomer structure.

The durability of the grafted FR treatments of flax fabrics will be studied in further investigations. Especially for textile applications, it is needed to assess the effect of the number of washing cycles and the washing temperature with tap water and detergents on the stability of the FR treatment and so on flame retardancy. Moreover, the effect of the radiation dose on the mechanical properties of the fabrics will be also evaluated.

\section{Acknowledgements}

The authors acknowledge Nicolas LE MOIGNE, associate professor from IMT - Mines Alès for his support on optical microscopy.

\section{Appendix A. Supplementary data}

Supplementary data to this article can be found online at https://doi.org/10.1016/j.polymdegradstab.2019.05.025.

\section{References}

[1] S. Sampaio, D. Bishop, J. Shen, Physical and chemical properties of flax fibres from stand-retted crops desiccated at different stages of maturity, Ind. Crops Prod. 21 (3) (2005) 275-284.

[2] J. Biagiotti, D. Puglia, J.M. Kenny, A review on natural fibre-based compositespart I: structure, processing and properties of vegetable fibres, J. Nat. Fibers 1 (2) (2004) 37-68

[3] D. Puglia, J. Biagiotti, J.M. Kenny, A review on natural fibre-based composites-Part II: application of natural reinforcements in composite materials fo automotive industry, J. Nat. Fibers 1 (3) (2005) 23-65.

[4] Y. Li, Q. Li, H. Ma, The voids formation mechanisms and their effects on the mechanical properties of flax fiber reinforced epoxy composites, Composites Part A 72 (2015) 40-48.

[5] M.E. Mngomezulu, M.J. John, V. Jacobs, A.S. Luyt, Review on flammability of biofibres and biocomposites, Carbohydr. Polym. 111 (2014) 149-182.

[6] S. Chapple, R. Anandjiwala, Flammability of natural fiber-reinforced composites and strategies for fire retardancy: a review, J. Thermoplast. Compos. Mater. 23 (6) (2010) 871-893.

[7] N.P.G. Suardana, M.S. Ku, J.K. Lim, Effects of diammonium phosphate on the flammability and mechanical properties of bio-composites, Mater. Des. 32 (4) (2011) 1990-1999.

[8] A.R. Horrocks, An introduction to the burning behaviour of cellulosic fibres
J. Soc. Dye. Colour. 99 (7-8) (1983) 191-197.

9] R.M. Kozlowski, M. Mackiewicz-Talarczyk, J. Barriga-Bedoya, Natural fibers production, processing, and application: inventory and future prospects, in: Contemporary Science of Polymeric Materials, vol. 1061, American Chemical Society, 2010, pp. 3-41.

[10] K. Opwis, A. Wego, T. Bahners, E. Schollmeyer, Permanent flame retardant finishing of textile materials by a photochemical immobilization of vinyl phosphonic acid, Polym. Degrad. Stabil. 96 (3) (2011) 393-395.

[11] M. Parvinzadeh Gashti, A. Almasian, UV radiation induced flame retardant cellulose fiber by using polyvinylphosphonic acid/carbon nanotube composite coating, Compos. B Eng. 45 (1) (2013) 282-289.

[12] M.J. Tsafack, J. Levalois-Grützmacher, Towards multifunctional surfaces using the plasma-induced graft-polymerization (PIGP) process: flame and waterproof cotton textiles, Surf. Coating. Technol. 201 (12) (2007) 5789-5795.

[13] J. Alongi, M. Ciobanu, G. Malucelli, Sol-gel treatments for enhancing flame retardancy and thermal stability of cotton fabrics: optimisation of the process and evaluation of the durability, Cellulose 18 (1) (Feb. 2011) 167-177.

[14] J.A. Harris, J.C. Arthur, W.R. Goynes, Flame resistant cotton fabrics prepared by radiation-initiated polymerization with vinyl phosphonate oligomer and $\mathrm{N}$ methylolacrylamide, J. Appl. Polym. Sci. 23 (9) (1979) 2555-2565.

[15] R. Sonnier, B. Otazaghine, A. Viretto, G. Apolinario, P. Ienny, Improving the flame retardancy of flax fabrics by radiation grafting of phosphorus compounds, Eur. Polym. J. 68 (2015) 313-325.

[16] R. Hajj, et al., Grafting of phosphorus flame retardants on flax fabrics: comparison between two routes, Polym. Degrad. Stabil. 147 (Jan. 2018) 25-34.

[17] M. Teixeira, et al., "Radiation-grafting of flame retardants on flax fabrics - a comparison between different flame retardant structures, Radiat. Phys. Chem. 145 (2018) 135-142.

[18] S. Gaan, G. Sun, Effect of phosphorus and nitrogen on flame retardant cellulose: a study of phosphorus compounds, J. Anal. Appl. Pyrolysis 78 (2) (Mar. 2007) $371-377$.

[19] L. Tibiletti, L. Ferry, C. Longuet, A. Mas, J.-J. Robin, J.-M. Lopez-Cuesta, Thermal degradation and fire behavior of thermoset resins modified with phosphorus containing styrene, Polym. Degrad. Stabil. 97 (12) (2012) 2602-2610.

[20] A.R.R. Horrocks, S. Zhang, Enhancing polymer flame retardancy by reaction with phosphorylated polyols. Part 2. Cellulose treated with a phosphonium salt urea condensate (Proban CC?) flame retardant, Fire Mater. 26 (4-5) (Jul. 2002) 173-182.

[21] S. Gaan, V. Salimova, P. Rupper, A. Ritter, H. Schmid, Flame Retardant Functional Textiles, Woodhead Publishing Limited, 2011.

[22] B. Schartel, Phosphorus-based flame retardancy mechanisms-old hat or a starting point for future development? Materials (Basel) 3 (10) (2010) 4710-4745.

[23] D. Price, et al., Flame retardance of poly(methyl methacrylate) modified with phosphorus-containing compounds, Polym. Degrad. Stabil. 77 (2) (2002) 227-233.

[24] S. Liang, M. Neisius, H. Mispreuve, R. Naescher, S. Gaan, Flame retardancy and thermal decomposition of flexible polyurethane foams: structural influence of organophosphorus compounds, Polym. Degrad. Stabil. 97 (11) (2012) $2428-2440$.

[25] R. Ménard, C. Negrell-Guirao, L. Ferry, R. Sonnier, G. David, Synthesis of new flame-retardants by radical chain transfer copolymerization of glycidyl methacrylate and dimethoxy-phosphorylmethyl methacrylate, Eur. Polym. J. 57 (2014) 109-120.

[26] J.R. Ebdon, et al., Flame retardance in some polystyrenes and poly(methyl methacrylate)s with covalently bound phosphorus-containing groups: initial screening experiments and some laser pyrolysis mechanistic studies, Polym. Degrad. Stabil. 69 (3) (2000) 267-277.

[27] L. Ferry, G. Dorez, a. Taguet, B. Otazaghine, J.M. Lopez-Cuesta, Chemical modification of lignin by phosphorus molecules to improve the fire behavior of polybutylene succinate, Polym. Degrad. Stabil. 113 (2015) 135-143.

[28] S.Y. Lu, I. Hamerton, Recent developments in the chemistry of halogen-free flame retardant polymers, Prog. Polym. Sci. 27 (8) (2002) 1661-1712.

[29] A.R. Horrocks, S. Zhang, Enhancing polymer char formation by reaction with phosphorylated polyols. 1. Cellulose, Polymer (Guildf) 42 (2001) 8025-8033.

[30] G. Dorez, B. Otazaghine, A. Taguet, L. Ferry, J.M. Lopez-Cuesta, Use of Py-GC/ MS and PCFC to characterize the surface modification of flax fibres, J. Anal. Appl. Pyrolysis 105 (2014) 122-130.

[31] G. Rosace, C. Colleoni, V. Trovato, G. Iacono, G. Malucelli, Vinylphosphonic acid/methacrylamide system as a durable intumescent flame retardant for cotton fabric, Cellulose 24 (7) (2017) 3095-3108.

[32] K. Williams, J.R. Ebdon, B.K. Kandola, Intumescent fire-retardant coatings for plastics based on poly(vinylphosphonic acid): improving water resistance with comonomers, J. Appl. Polym. Sci. 47601 (2019) 1-11.

[33] C. Huggett, Estimation of rate of heat release by means of oxygen consumption measurements, Fire Mater. 4 (2) (1980) 61-65.

[34] N. Hernandez, R. Sonnier, S. Giraud, Influence of grammage on heat release rate of polypropylene fabrics, J. Fire Sci. 36 (1) (2018) 30-46.

[35] M.I. Nelson, Ignition mechanisms of thermally thin thermoplastics in the cone calorimeter, Proc. R. Soc. London. Ser. A Math. Phys. Eng. Sci. 454 (1971) 789-814. Mar. 1998.

[36] E. Mikkola, I.S. Wichman, On the thermal ignition of combustible materials, Fire Mater. 14 (3) (1989) 87-96.

[37] V. Babrauskas, W.J. Parker, Ignitability measurements with the cone calorimeter, Fire Mater. 11 (1) (1987) 31-43. 
[38] J. Tata, J. Alongi, F. Carosio, A. Frache, Optimization of the procedure to burn textile fabrics by cone calorimeter: Part I. Combustion behavior of polyester. Fire Mater. 35 (6) (Oct. 2011) 397-409.

[39] S. Bebe, Estimation of Free Radical Polymerization Rate Coefficients Using Computational Chemistry, Queen's University, 2008.

[40] G. David, C. Negrell-guirao, I. Charles, G. Icgm, E. Inge, Phosphorus-Containing Vinyl or Allyl Monomers, no. 11, 2014.
[41] R. Sonnier, L. Ferry, J.-M. Lopez-Cuesta, CHAPTER 12. Flame retardancy of phosphorus-containing polymers, in: S. Monge, G. David (Eds.), Phosphorusbased Polymers. From Synthesis to Applications, vol. 11, 2014, pp. 252-270. Cambridge.

[42] B. Schartel, T.R. Hull, Development of fire-retarded materials-interpretation of cone calorimeter data, Fire Mater. 31 (5) (2007) 327-354. 